\title{
Positional obstructive sleep apnea as a new interdisciplinary challenge
}

\author{
M. Milkov + \\ Department of Dental Materials Science and \\ Propaedeutics of Prosthetic Dental Medicine, \\ Faculty of Dental Medicine, \\ Professor Paraskev Stoyanov Medical University of Varna
}

\begin{abstract}
Severe obstructive sleep apnea (OSA) is a life-threatening condition in children and adults. Recently, a rising interest emerges in the positional OSA (POSA) as a specific clinical manifestation a relatively common sleep breathing disorder in young and adult patients. In the present concise survey, some essential characteristics of POSA reported in the newly published literature are described. A special attention is paid to the social epidemiology, diagnosis, and modern treatment of POSA from an interdisciplinary point of view.
\end{abstract}

Key words: positional obstructive sleep apnea, epidemiology, diagnosis, treatment

Running head: Positional obstructive sleep apnea - a new challenge

\section{Definition}

Positional sleep apnea is functionally defined as a total apnea-hypopnea index $\geq 5$ with a $>50 \%$ apneahypopnea index reduction between the supine and nonsupine postures, and an apnea-hypopnea index that normalizes down to $<5$ in the nonsupine posture (1).

\section{Positional obstructive sleep apnea classifications}

Four criteria for the positional obstructive sleep apnea (POSA) such as Amsterdam Positional OSA Classification (APOC), supine apnea-hypopnea index twice the non-supine apnea-hypopnea index (2), Cartwright plus the non-supine apnea-hypopnea index $<5(1)$, and the overall apnea-hypopnea index severity at least 1,4 times the non-supine severity based on American Academy Sleep Medicine (AASM) 2007 and 2012 hypopnea scoring definitions are systematically compared by using 142 in-home polysomnography records (3). The prevalence of POSA based on the Cartwright and the overall apnea-hypopnea index severity criteria is approximately $60 \%$ in those with at least mild OSA by apnea-hypopnea index 2007 and apneahypopnea index 2012. A POSA prevalence reduction by $16 \%$ for apnea-hypopnea index 2012 versus apnea-hypopnea index 2007 is attributed to higher mild OSA incidence.

Both Cartwright and overall apnea-hypopnea index severity criteria for POSA based on AASM 2007 
and 2012 hypopnea scoring definitions exhibit the strongest sensitivity while both overall apneahypopnea index severity and Cartwright plus the non-supine apnea-hypopnea index $<5$ criteria for POSA based on these definitions present with the strongest specificity for identification of the patients expected to have sleep disordered breathing severity reduction by $25 \%$ or $35 \%$ with positional therapy (3).

In a retrospective, single-centre cohort study of 253 OSA patients, there is a prevalence of POSA of $69 \%$ when applying APOC and of $64 \%$ when applying Cartwright's classification (4). There are inverse correlations between POSA and body mass index as well as between POSA and apnea-hypopnea index.

\section{Epidemiology of POSA}

In a retrospective study of 326 patients, there is POSA in 49 of 99 patients (in 49,49\%) with mild, 14 of 72 patients (in $19,44 \%$ ) with moderate, and 5 of 77 patients (in $6,49 \%$ of the cases) with severe OSA (1).

Among 279 consecutive patients with OSA, 216 subjects $(77,42 \%$ of the cases $)$ meet the criteria for POSA (5). Of them, 158 (73,15\%) present with supine-predominant OSA and the rest 58 subjects (26,85\% of the cases) do with supine-isolated OSA. The latter have lower arousal indices, but poorer quality of sleep, and are more depressed and anxious when compared with the supine-predominant OSA subjects.

The relative share of POSA patients among OSA ones varies in the recent literature available, e.g. 67 out of $120(55,83 \%)(6), 321$ out of $574(55,92 \%)$ (7), 96 out of 144 OSA patients $(66,67 \%)$ (8) as well as 733 out of 1184 supine predominant patients $(61,91 \%)(9)$. The average time in supine position is by 37 min longer in the POSA group than in non-POSA one.

In the population-based HypnoLaus Sleep Cohort in Switzerland, POSA is detected in 918 out of 1224 OSA subjects aged $40-85$ years (in $75,00 \%$ ), while there is exclusive POSA in 441 of these 1224 OSA ones (in $36,03 \%$ of the cases) (10). In multivariate analyses, lower apnea-hyponea index and lower body mass index are associated with POSA and exclusive POSA in males.

Within a 52-month retrospective study based on self-administered home-based respiratory polygraphy records, a prevalence of POSA of 54,6\% among
OSA patients is established (11). The patients with POSA are younger, have a statistically reliably lower body mass index $(30,3 \pm 0,9$ versus $35,3 \pm 1,2$ $\left.\mathrm{kg} / \mathrm{m}^{2} ; \mathrm{p}<0,0001\right)$, and spend less time with oxygen saturation $<90 \%(8,8$ versus $28,7 \pm 6,7 ; \mathrm{p}=$ $0,0038)$. These patients spend $43 \%$ of total recording time in the supine position.

Several authors agree with this generalization concerning POSA patients' features, too $(6,7,9)$. Besides POSA patients present with a less severe OSA.

\section{Diagnosis of POSA}

Snoring severity index, clinical nasal obstruction, septal deviation, conchal hypertrophy, and allergic rhinitis scores, overall apnea-hypopnea index and apnea-hypopnea index in supine and nonsupine positions, daytime sleepiness scores, and body mass index are comparatively evaluated in 44 male OSA patients at a mean age of $48,0 \pm 6,8$ years (range 31 to 60 years), 22 with POSA and 22 with non-POSA (12). The apnea-hypopnea index is statistically significantly lower in POSA than in non-POSA males $(p<0,03)$. Spearman correlation analysis revealed There is a statistically significant negative correlation between allergic rhinitis score and POSA (Spearman coefficient of $r=-0,40 ; p<$ $0,0001)$ as well as a positive correlation between the apnea-hypopnea index and body mass index (Pearson coefficient of $r=0,32 ; p<0,05$ ).

The result from a comparative retrospective study of 30 POSA and 45 non-POSA patients by means of polysomnography and computed tomography demonstrate statistically significant differences $(p$ $<0,05$ ) between both groups in terms of the apneahypopnea index, lateral apnea-hypopnea index, soft palate length, cross-sectional palatopharyngeal area, and coronal diameter of the palatopharyngeal area at the narrowest glossopharyngeal part (13). Only the apnea-hypopnea index, lateral apneahypopnea index and soft palate length values are lower in POSA patients. The lateral apnea-hypopnea index correlates statistically significantly with the cross-sectional area $(r=-0,306 ; p=0,008)$ and coronal diameter $(r=-0,398 ; p<0,001)$ of the palatopharyngeal area, the cross-sectional area $(\mathrm{r}=$ $-0,241 ; p=0,038)$ and coronal diameter $(r=-0,297$; $\mathrm{p}=0,010)$ of the narrowest glossopharyngeal part, the coronal glossopharyngeal diameter $(r=0,284$; $\mathrm{p}=0,013)$, body mass index $(\mathrm{r}=0,273 ; \mathrm{p}=0,018)$, soft palate length $(\mathrm{r}=0,284 ; \mathrm{p}=0,014)$, and val- 
lecula-tip of tongue $(r=0,250 ; p=0,030)$ as well. The prevalence rate of POSA in 1052 Korean OSA adults is $75,6 \%$ with $39,9 \%$ having overall apneahypopnea index normalized to $<5$ /hour while in non-supine position (14). POSA patients have milder OSA, older age, and lower body mass index than non-POSA ones as POSA does not influence upon daytime sleepiness, depressive symptoms, anxiety, and health-related quality of life. There are statistically significant differences in supine sleep time depending on the positional tendency of OSA.

The retrospective study of 171 patients with obstructive sleep apnea hypopnea syndrome (OSAHS) in Beijing Tongren Hospital, Capital Medical University, Beijing, China, between July 2012 and June 2014 identifies 47 patients $(27,495 \%$ of the cases) with P-OSAHS (15). There are significant differences between POSAHS patients and non-POSAHS ones in terms of body mass index, neck circumference, apnea-hypopnea index, lowest oxygen saturation, percentage of time with oxygen saturation below $90 \%$, and minimal anterior-posterior airway glossopharyngeal dimension $(\mathrm{p}<0,05)$. The apnea-hypopnea index is the only predictive parameter for P-OSAHS $(\mathrm{p}<0,01)$. The incidence of P-OSAHS differs statistically significantly between patients with mild or moderate, severe and extremely severe disease $(81,82 \%$ versus $45,28 \%$ versus $5,21 \%$; $p<0,05$ ).

In a retrospective study in Japan, 643 obstructive sleep apnea syndrome (OSAS) patients are categorized as positional or non-positional patients based on the following criteria: age, body mass index, arterial hypertension, tongue enlargement, palatine tonsil hypertrophy, pharyngeal tonsil hypertrophy, obstruction by Muller's maneuver, and polysomnography (16). The patients with body mass index $<25 \mathrm{~kg} / \mathrm{m}^{2}$ are clearly more frequent among positional than among non-positional OSAS and present more commonly with tongue enlargement, palatine tonsil hypertrophy and obstruction by Muller's maneuver.

Within a cross sectional study of 278 OSA patients, 147 patients present with POSA $(52,87 \%$ of the cases) (17). These patients are statistically significantly younger $(p=0,005)$, have lower body mass index $(\mathrm{p}=0,0001)$, lower prevalence of arterial hypertension $(\mathrm{p}=0,006)$, lower Berlin $(\mathrm{p}=0,01)$ and STOP $(p=0,001)$ questionnaire scores as well as smaller neck $(\mathrm{p}=0,005)$ and waist circumferences $(p=0,009)$ than non-POSA patients.

\section{Recent advances in POSA treatment}

Recent technological advances renew interest in positional therapy because of the invention of new sophisticated vibratory positional therapy devices (18). These new devices show great promise with efficacy and markedly improve patient's tolerance and long-term adherence.

The NightBalance Sleep Position Trainer builds up vibrations during the positional therapy in a cohort of 51 consecutive patients with POSA (19). Of them, only 27 patients use the device throughout the long-term period. The baseline percentaghe of supine is lower than during polysomnography $(22,9 \pm 16,2$ versus $40,7 \pm 20,0 \% ; p=0,0005)$. It drops on short term $(\mathrm{p}=0,0001)$ without further change on long term. Apnea-hypopnoea index calculation reveals that two patients would not have OSA at home. Ten patients are cured and demonstrate higher response on vibrations. Only 29 patients are adherent and show higher response on vibrations and less side effects.

The efficacy of a sleep position trainer is assessed by polysomnography in 58 patients with positional obstructive sleep apnea during an one-year period (20). Nine patients (15,52\% of the cases) stop using the this device. After one year, polysomnography performed in 34 middle-aged and overweight patients with overall apnea/hypopnea index of $16 /$ hour reveals a statistically significant reduction down to $6 /$ hour $(p<0,001)$. The median percentage of supine sleep decreases statistically significantly to $1 \%(\mathrm{p}<0,001)$. The mean objective device use in 28 patients is $7,3 \pm 0, .9$ hours/night and $69 \pm 26 \%$ of the nights. Since the start of sleep position trainer treatment, a better patient's sleep quality is reported by $75 \%$ of the cases.

In 2012, drug-induced sleep endoscopy is performed in 424 OSA patients of whom 257 are positional and 167 are non-positional patients (21). The positional patients are younger, have smaller neck circumference, lower body mss index and apneahypopnea index than the non-positional ones. The complete concentric collapse is less common in the positional patients (in $31,5 \%$ ) as compared to the non-positional ones (in $46,1 \%$ of the cases). After upper airway surgery, OSA often is cured or improved to less severe POSA, however, surgical efficacy is lower in the positional patients than in the non-positional ones.

The effect of some passive maneuvers such as positional therapy, oral appliance therapy, and 
combined treatment on upper airway patency during drug-induced sleep endoscopy and on treatment outcomes is evaluated in 200 OSA patients at a mean age of $50,1 \pm 11,7$ years, with a body mass index of $27,0 \pm 3,1 \mathrm{~kg} / \mathrm{m}^{2}$ and a median apnea-hypopnea index of 19,2/hour (22). Of them, 112 patients present with POSA, 38 of the latter $(33,93 \%)$ - with supine isolated and $74(66,07 \%$ of the cases) - with supine predominant POSA. Lateral head rotation reduces POSA by $33,3 \%$ in supine predominant and by $50 \%$ in supine isolated POSA patients. Combining the maneuvers of manual jaw thrust with lateral head rotation results in a reduction by more than $75 \%$ in all the patients.

Positional therapy of 28 POSA patients is carried out during one month by means of a sleep-positioning pillow (Posiform ${ }^{\circledR}$ ) in a prospective cohort study (23). There are statistically significant immediate treatment effects after one night which sustain after one month being characterized by significant reductions of sleep in supine position $(\mathrm{p}<0,001)$, sleep fragmentation $(\mathrm{p}<0,05)$, apnea-hypopnea $(\mathrm{p}<0,001)$, respiratory disturbance $(\mathrm{p}<0,001)$ and oxygen desaturation $(\mathrm{p}<0,001)$ indices as well as significant and persistent improvements of the Pittsburgh Sleep Quality Index $(p<0,001)$, the Epworth Sleepiness Scale $(p<0,005)$ and the Function Outcomes of Sleep Questionnaire $(\mathrm{p}<$ 0,001).

A literature search in EMBASE since 2012 identifies ten studies demonstrating the effectiveness of positional therapy in POSA patients (24). Three publications propose different subclassification systems for POSA. There are cost benefits of incorporating this effective therapy into OSA management. Progress has been made in development of tools for measuring compliance. Creating a subclassification of POSA helps developing targeted therapy for patients and determining its use as standalone or adjunct therapy.

In conclusion, POSA represents a true interdisciplinary challenge for both modern theoretical sleep medicine research and clinical practice in adults. United efforts by rhinologists, pulmologists, neurologists, pharmacologists, radiologists as well as by sleep medicine specialists are needed.

\section{References}

1. Mador MJ, Kufel TJ, Magalang UJ, Rajesh SK, Watwe V, Grant BJ. Prevalence of positional sleep apnea in patients undergoing polysomnography. Chest 2005;28: 2130-2137.

2. Cartwright RD, Diaz F, Lloyd S. The effects of sleep posture and sleep stage on apnea frequency. Sleep 1991; 14: 351-353.

3. Levendowski DJ, Oksenberg A, Vicini C, Penzel T, Levi M, Westbrook PR. A systematic comparison of factors that could impact treatment recommendations for patients with Positional Obstructive Sleep Apnea (POSA). Sleep Med 2018; 50: 145-151.

4. Ravesloot MJ, Frank MH, van Maanen JP, Verhagen EA, de Lange J, de Vries N. Positional OSA part 2: retrospective cohort analysis with a new classification system (APOC). Sleep Breath 2016; 20: 881-888.

5. Kim KT, Cho YW, Kim DE, Hwang SH, Song ML, Motamedi GK. Two subtypes of positional obstructive sleep apnea: Supine-predominant and supine-isolated. Clin Neurophysiol 2016; 127: 565-570.

6. Richard W, Kox D, den Herder C, Laman M, van Tinteren H, de Vries N. The role of sleep position in obstructive sleep apnea syndrome. Eur Arch Otorhinolaryngol 2006; 263: 946-950.

7. Oksenberg A, Silverberg DS, Arons E, Radwan H. Positional vs nonpositional obstructive sleep apnea patients: anthropomorphic, nocturnal polysomnographic, and multiple sleep latency test data. Chest 1997; 112: 629-639.

8. Teerapraipruk B, Chirakalwasan N, Simon R, et al. Clinical and polysomnographic data of positional sleep apnea and its predictors. Sleep Breath 2012; 16: 1167-1172.

9. Joosten SA, Hamza K, Sands S, Turton A, Berger P, Hamilton G. Phenotypes of patients with mild to moderate obstructive sleep apnoea as confirmed by cluster analysis. Respirology 2012; 17: 99-107.

10. Heinzer R, Petitpierre NJ, Marti-Soler H, Haba-Rubio J. Prevalence and characteristics of positional sleep apnea in the HypnoLaus population-based cohort. Sleep Med 2018; 48: 157-162.

11. Di-Tullio F, Ernst G, Robaina G, et al. Ambulatory positional obstructive sleep apnea syndrome. Sleep Sci 2018; 11: 8-11.

12. Cevizci R, Kemaloğlu YK, Yılmaz M, Düzlü M, Karamert R. Role of nasal problems on positional and nonpositional obstructive sleep apnea. Kulak Burun Bogaz Ihtis Derg 2016; 26: 219-324.

13. Jiao X, Zou J, Liu S, Guan J, Yi H, Yin S. A retrospective study: does upper airway morphology differ between non-positional and positional obstructive sleep apnea? Peer J 2017 Oct 13;5:e3918. doi: 10.7717/peerj.3918

14. Lee SA, Paek JH, Chung YS, Kim WS. Clinical features in patients with positional obstructive sleep apnea according to its subtypes. Sleep Breath 2017; 21: 109-117.

15. Sun N, Ye JY, Ni X, et al. Evaluation of the clinical characteristics of patients with positional obstructive sleep apnea hypopnea syndrome. Zhonghua Er Bi Yan Hou Tou Jing Wai Ke Za Zhi 2016; 51: 801-805 (in Chinese).

16. Endo M, Kondo T, Shimada R, Tsukahara K. Decision making for position-dependent obstructive sleep apnea syndrome on the basis of patient information and physical examinations of the upper airway, acquired on an outpatient basis. Acta Otolaryngol 2018; 138: 562-566.

17. Oulhaj A, Al Dhaheri S, Su BB, Al-Houqani M. Discriminating between positional and non-positional obstructive sleep apnea using some clinical characteristics. Sleep Breath 2017; 21: 877-884. 
18. Yingjuan M, Siang WH, Leong Alvin TK, Poh HP. Positional therapy for positional obstructive sleep apnea. Sleep Med Clin 2019;14(1):119133.

19. Buyse B, Ciordas S, Hoet F, Belge C, Testelmans D. Positional obstructive sleep apnoea: challenging findings in consecutive patients treated with a vibrating position trainer. Acta Clin Belg 2018 Nov 15:1-9. doi: 10.1080/17843286.2018.1545374

20. Beyers J, Vanderveken OM, Kastoer C, Boudewyns A, De Volder I, Van Gastel A, et al. Treatment of sleep-disordered breathing with positional therapy: long-term results. Sleep Breath 2019 Feb 18. doi: 10.1007/s11325-019-01792-9

21. Kastoer C, Benoist LBL, Dieltjens M, Torensma B, de Vries LH, Vonk PE, et al. Comparison of upper airway collapse patterns and its clinical significance: drug-induced sleep endoscopy in patients without obstructive sleep apnea, positional and non-positional obstructive sleep apnea. Sleep Breath. 2018; 22: 939-948.

22. Vonk PE, Beelen AMEH, de Vries N. Towards a prediction model for drug-induced sleep endoscopy as selection tool for oral appliance treatment and positional therapy in obstructive sleep apnea. Sleep Breath 2018; 22: 901-907.

23. Newell J, Mairesse O, Neu D. Can positional therapy be simple, effective and well tolerated all together? A prospective study on treatment response and compliance in positional sleep apnea with a positioning pillow. Sleep Breath 2018; 22: 1143-1151.

24. Omobomi O, Quan SF. Positional therapy in the management of positional obstructive sleep apnea - a review of the current literature. Sleep Breath 2018; 20: 297-304.

\section{Corresponding author:}

Assoc. Prof. Mario Milkov, MD, PhD

Department of Dental Materials Science and

Propaedeutics of Prosthetic Dental Medicine, Faculty of Dental Medicine

Professor Paraskev Stoyanov Medical University of Varna 100 Tsar Osvobodite Blvd

Varna 9002

Bulgaria

Phone: +359-888 897403

E-mail:mario.milkov@gmail.com 